ACTA UNIVERSITATIS LODZIENSIS

Folia Litteraria Romanica 14, 2019

http://dx.doi.org/10.18778/1505-9065.14.10

$c o p p$

\title{
Joanna Cholewa
}

Uniwersytet w Białymstoku

(iD) https://orcid.org/0000-0002-0545-8470

j.cholewa@uwb.edu.pl

\section{Constructions causatives avec le verbe boire : étude contrastive français/polonais}

\begin{abstract}
RÉSUMÉ
L'article prend pour objet la construction causative du français faire boire et ses correspondants en langue polonaise. Dans celle-ci, le prédicat causatif de base, exprimant le mieux ce sens causatif est le verbe poić, ainsi que ses dérivés préfixaux aspectuels, que l'on peut désigner par le symbole (-)poić. L'objectif de l'analyse est d'observer les régularités qui se manifestent au niveau du choix du verbe polonais au moment de la traduction de faire boire. L'analyse démontre que le polonais sélectionne différents verbes, selon les sens de faire boire, distingués sur la base de leur construction sémanticosyntaxique. Les emplois que l'on peut appeler prototypiques, se référant aux entités animées, sont traduits en polonais par poić / napoić. Cependant, pour traduire faire boire avec N1 appartenant à la classe [alcool], le polonais sélectionnera soit napoić (sans excès), soit spoić (excessivement). La présence du quantifieur en position de N1 bloque entièrement (-)poić. Enfin, la construction passive se faire boire se traduit par wsiąkać / wsiaknać, prédicat non causatif.
\end{abstract}

MOTS-CLÉS - construction causative, prédicat causatif, analyse contrastive, boire, poić

\section{Causative Constructions with the Verb 'boire': A French-Polish Contrastive Analysis}

\section{SUMMARY}

This paper analyses the French causative construction 'faire boire' and its equivalents in the Polish language. The basic causative predicate which translates the sense of faire boire is the verb 'poic' and its prefixal aspectual equivalents. The aim of the analysis is to observe the regularities that occur in the choice of the Polish verb at the moment of the translation of 'faire boire'. The analysis shows that, depending on the meaning of 'faire boire' - one distinguished on the basis of a semantic-syntactic construction - the Polish language selects different verbs. The use that we can call prototypical is translated into Polish with 'poić/napoic', referring to both humans and animals. However, if N1 belongs to the class [alcohol], the Polish language selects either 'napoic' (meaning: not excessively), or 'spoić' (meaning: in excess). The presence of the quantifier in position N1 blocks the use of the verb ' $p o i c$ '. The French passive construction 'se faire boire' can be translated with the predicate 'wsiakać / wsiaknac', showing no causativity.

KEYWORDS - causative construction, causative predicate, contrastive analysis 


\section{Introduction}

Considéré soit comme un semi-auxiliaire, soit comme un auxiliaire, faire se construit avec de nombreux verbes, créant ainsi des constructions appelées causatives ou factitives. Comme le constate Shyldkrot ${ }^{1}$, la différenciation entre les uns et les autres n'est ni univoque ni communément admise. D'après Riegel, il est appelé 'auxiliaire causatif' et sa construction avec un verbe à l'infinitif - construction 'causative' ou 'factitive'. Le sujet de faire dans cette construction est actif et représente la cause du procès exprimé par la structure infinitive. Le classement de faire dans le groupe des auxiliaires se justifie de la façon suivante : " On les enregistre comme auxiliaires dans la mesure où, comme être et avoir, leur sémantisme se réduit à une indication grammaticale (à la différence de verbes de sens « plein ») et où la construction auxiliaire + infinitif semble bien parallèle à la construction avoir / être + participe passé $»^{2}$. Riegel distingue aussi la construction se faire + infinitif, où se faire est un auxiliaire de passivation, mais garde sa valeur causative, en impliquant de la part du sujet un certain degré de responsabilité ${ }^{3}$. Grevisse maintient la distinction traditionnelle entre être et avoir d'une part (qu'il nomme auxiliaires) et les autres verbes comme laisser, faire, paraître, sembler, risquer, savoir (les semi-auxiliaires). Selon lui, faire est un semi-auxiliaire qui «sert à former une périphrase factitive, de sens causatif $»^{4}$.

Ponchon $^{5}$ considère faire comme auxiliaire de causation, en expliquant la différence entre les constructions infinitives avec ce verbe et avec d'autres, par exemple entendre de la façon suivante. Si l'on compare deux phrases : Max fait chanter Luc et Max entend chanter Luc, on s'aperçoit que, dans la deuxième, il existe deux actions concomittantes, indépendantes : Max entend et Luc chante. Or, dans la première, où Max est sujet, fait verbe prédicatif et chanter Luc objet (dans lequel Luc est sujet de l'auxilié infinitif chanter), il existe une relation de dépendance du second groupe complément par rapport à faire. Nous avons affaire à une subordination des actions. La construction de deux phrases indépendantes Max fait et Luc chante est impossible.

Pour $\mathrm{Gross}^{6}$, les constructions faire + infinitif sont une combinaison de l'opérateur causatif, appliqué à une phrase élémentaire :

\footnotetext{
${ }^{1}$ H. Bat-Zeev Shyldkrot, "Présentation. Les auxiliaires : délimitation, grammaticalisation et analyse », Langages, $\mathrm{n}^{\circ} 135,1999$, p. 3.

${ }^{2}$ M. Riegel, Grammaire méthodique du français, Paris, Presses Universitaires de France, 1994, p. 451.

3 Ibid., p. 742-743.

${ }^{4}$ M. Grevisse, Le Bon usage, Louvain-la-Nauve, Duculot, 1993, p. 1234.

${ }_{5}^{5}$ T. Ponchon, Sémantique lexicale et sémantique grammaticale: le verbe 'faire' en français médiéval, Genève, Droz, 1994, p. 185-186.

${ }^{6}$ M. Gross, «Les bases empiriques de la notion de prédicat sémantique », Langages, 1981, n 63, p. 24.
} 
Max fait dormir Luc $\rightarrow$ Max fait \# Luc dort

Max fait boire du vin à Luc $\rightarrow$ Max fait \# Luc boit du vin.

En polonais, la causativité peut s'exprimer à l'aide des constructions analytiques avec des verbes opérateurs? ${ }^{7}$ par exemple nadawać / nadać giętkość ('ajouter de la souplesse'), powodować / spowodować zmęczenie ('causer la fatigue'). Il existe aussi des prédicats causatifs, comme par exemple ujędrnić ('rendre ferme'), utwardzić ('rendre dur'). La présente analyse prendra pour objet la construction faire boire, ainsi que le verbe polonais poić et certains de ses dérivés. Ce verbe est un prédicat causatif que l'on peut, certes, paraphraser par dawać / dać pić, dawać / dać do picia, mais la possibilité d'une telle paraphrase n'est pas systématiquement possible dans tous les emplois. Le but de l'analyse est d'observer les régularités qui se manifestent au niveau du choix du verbe polonais au moment de la traduction de faire boire, utilisé sans complément ou avec celui-ci, et possédant alors des caractéristiques sémantiques variées.

\section{Boire et (-)pić}

Pour commencer notre analyse, nous voulons consacrer quelques réflexions au verbe boire, base de la construction factitive faire boire, et son correspondant polonais (-)pić, le signe (-) symbolisant chacun des préfixes possibles.

Le verbe transitif boire signifie (selon $T L F i^{8}$ ):

1. avec le sujet désignant une personne ou un animal, 'avaler un liquide' :

a) le complément direct est exprimé : boire de l'eau, un verre ;

b) dans l'emploi absolu :

- le complément non exprimé peut désigner toute espèce de boissons : boire chaud, frais, à longs traits ; boire à sa soif ; boire dans un verre ; verser à boire ;

- boire du vin ou des boissons alcoolisées ; avoir coutume d'en boire avec excès, être alcoolique ;

c) par analogie, avec le sujet désignant un corps perméable ou poreux, 'absorber un liquide ; se laisser pénétrer, imprégner par lui';

2. au sens figuré, avec le sujet désignant généralement une personne :

a) 'recevoir un bien d'ordre physique, moral ou intellectuel et en jouir ou en tirer parti intensément' : C'est à la vraie source de sa vie que son âme va boire;

b) 'surmonter une difficulté' : Cheval qui boit l'obstacle 'qui le franchit très facilement';

\footnotetext{
7 A. Zatorska, «Z Z problematyki polskich i słoweńskich analityzmów kauzatywnych z parafrazą przymiotnikową », Studia z Filologii Polskiej i Stowiańskiej, 2015, nº 50, p. 237-248.

8 Trésor de la Langue Française informatisé, http://atilf.atilf.fr/.
} 
c) 'supporter quelque chose de pénible, d'humiliant' : Boire l'amertume, un affront, la honte.

Le dictionnaire Les Verbes français $\left(L V F^{9}\right)$ délimite et divise différemment les sens de boire. Il en distingue quatre, dont un est un emploi passif:

boire 1 - 'chopiner, zinguer' : On boit de l'eau, Ce vin se boit facilement, On boit beaucoup l'été ;

boire 2 - 'se soûler' : On boit depuis l'adolescence, On boit de l'alcool ;

boire 3 - 's'imbiber, s'imprégner' : Cette éponge boit bien, Le buvard boit l'encre;

boire 4 (être bu) - 'être ivre' : On est bu après cette réunion.

Nous pouvons constater que $L V F$ fait la différence entre 'boire un peu, n'importe quelle boisson' (boire 1) et 'boire excessivement, en parlant de l'alcool, se soûler' (boire 2), emplois qui sont confondus dans TLFi. Par contre, LVF confond les emplois avec le complément d'objet direct exprimé et les emplois absolus, qui figurent à part dans $T L F i$.

Le correspondant polonais de boire est le verbe imperfectif pić, auquel s'ajoutent de nombreux préverbés perfectifs : wypić, podpić, upić, napić się, spić, dopić, sélectionnés en fonction de la valeur aspectuelle que l'on voudrait exprimer. Dans les langues slaves, l'adjonction d'un préfixe entraîne, en général, le changement de l'aspect grammatical du verbe de base ${ }^{10}$, un verbe imperfectif devient perfectif. À cela s'ajoutent les variations dites aspectuelles : «Il s'agit de la façon dont le procès se déroule, dont il occupe le temps $\rangle^{11}$. C'est ce dernier sens que prend le mot 'aspect' par rapport à la langue française. Les variations aspectuelles sont souvent exprimées par le préfixe du verbe polonais, qui n'a pas que la fonction de perfectivation.

Pić s'utilise dans les sens suivants $\left(W S J P^{12}\right)$ :

1. 'nabierać płyn do ust i połykać go' ('prendre du liquide dans sa bouche et l'avaler') : pić herbatę, kawę; wódkę, piwo ('boire du thé, du café ; de la vodka, de la bière');

2. 'spożywać alkohol, zwłaszcza w nadmiarze' ('consommer de l'alcool, surtout excessivement') : mąż, ojciec pije ; pić w barze, w pracy ; pić z kolegami ('le mari, le père boit ; boire dans un bar, au travail ; boire avec des copains') ;

3. 'wciągać płyn do wnętrza' ('absorber du liquide') : kwiaty, rośliny pija ('les fleurs, les plantes boivent') ;

\footnotetext{
${ }^{9}$ Les Verbes français de Jean Dubois et Françoise Dubois-Charlier (Version LVF+1), http://rali.iro. umontreal.ca/rali/?q=fr/lvf.

${ }^{10}$ D. Stosic, « Le rôle des préfixes dans l'expression du déplacement. Eléments d'analyse à partir des données du serbo-croate et du français ", Cahiers de Grammaire, 2001, n 26, p. 207-228, $<$ halshs-00272879>, consulté le 21.05.2018.

${ }^{11}$ O. Ducrot et J.-M. Schaeffer, Nouveau dictionnaire encyclopédique des sciences du langage, Paris, Éditions du Seuil, 1995, p. 691.

12 Wielki Słownik Języka Polskiego, http://www.wsjp.p1/.
} 
4. 'być zbyt ciasnym lub niewygodnym' ('être trop étroit ou incommode') : marynarka pije; buty pija; pić pod pachami ('le veston est trop serré, les chaussures font mal, qch serre les aisselles') ;

5. 'książk. robić aluzje' (littéraire, 'faire des allusions').

\section{Faire boire et (-)poić}

Dans la construction faire boire, boire apparaît dans presque tous ses sens, y compris figurés, à l'exception du sens 'absorber un liquide', où le sujet est inanimé. Faire boire se traduit en polonais par plusieurs verbes et locutions verbales. La solution la plus évidente est d'utiliser le verbe causatif poić. Le dictionnaire WSJP en propose les définitions suivantes :

1) 'przygotowywać i podawać napój' ('préparer et servir une boisson') : poić dziecko, chorego ; zwierzęta ; poić alkoholem, herbata, mlekiem ('faire boire l'enfant, le malade ; les animaux ; faire boire de l'alcool, du thé, du lait') ;

2) 'książk. odczuwać przyjemność, odbierając jakieś bodźce' (littéraire 'ressentir un plaisir, en captant des stimuli') : poić oczy, uszy ; poić cisza (faire boire les yeux, les oreilles ; faire boire du silence) ;

3) sprawiać, że ktoś w nadmiarze spożywa alkohol ('faire que quelqu'un consomme trop d'alcool) : poić piwem, wódka ('faire boire de la bière, de la vodka').

Les définitions du dictionnaire $P W N^{13}$ ne sont pas identiques :

1) 'dawać komuś, czemuś pić, aż do ugaszenia pragnienia' ('donner à boire à quelqu'un jusqu'à assouvir sa soif') ;

2) 'dawać komuś do picia alkohol' ('donner à quelqu'un à boire de l'alcool') ;

3) 'zmuszać kogoś do wypicia czegoś' ('forcer quelqu'un à boire quelque chose') ;

4) 'wywoływać w kimś jakieś uczucie' ('provoquer en quelqu'un un sentiment quelconque').

Rien que sur la base de ces deux dictionnaires, nous remarquons une incertitude, voire une confusion des sens de poić. D'un côté, WSJP met ensemble, dans le sens 1 ('préparer et servir une boisson'), toutes sortes de liquides (eau, lait, thé, alcool), alors que $P W N$ distingue un sens à part si le complément d'objet est l'alcool (sens 2 : 'donner à quelqu'un à boire de l'alcool'). En revanche, quand PWN met ensemble les emplois avec le complément 'toutes sortes de liquides' (sens 1), il y ajoute en plus 'à sa soif'. Ce dictionnaire distingue encore 'forcer à boire' (sens 3), et un sens figuré se référant aux sentiments (sens 4).

Le verbe poić, tout comme pić, forme également des préverbés, dont chacun à un sens spécifique, par exemple spoić se réfère uniquement à l'alcool $(P W N$ : 'upić kogoś' - 'soûler qqn'), alors que napoić est beaucoup plus général ( $P W N$ :

13 Stownik Języka Polskiego PWN, https://sjp.pwn.pl/. 
'dać komuś coś do picia' - 'donner à boire à quelqu'un'). Il est bien visible que chacune de ces formes perfectives se réfère à un autre sens de l'imperfectif poić : spoić au sens 2 et napoić au sens 1. Le fait que différents préverbés perfectifs se réfèrent à des sens particuliers de poić a été probablement à la base de la distinction faite par $P W N$ des sens 1 et 2 . Nous avons ainsi des couples imperfectif/perfectif poić / napoić (pour le sens 1 de poić) et poić / spoić (pour le sens 2).

Notre objectif sera maintenant de voir quels verbes sélectionne la langue polonaise pour traduire différents emplois de faire boire.

\section{Structures avec faire boire}

\section{a. $X$ fait boire $+N_{\text {1/liquidel }} \grave{a} \mathbf{Y}$}

La première structure envisagée est $X$ fait boire $+N_{\text {I[liquide] }} \grave{a} Y$. Le correspondant polonais le plus évident de ce sens de faire boire est le couple poić / napoić, où la première forme exprime l'inachèvement et la deuxième, préfixale, l'achèvement. Les deux traduisent le verbe faire boire, utilisé avec le nom massif, précédé de l'article partitif, défini ou indéfini. Ainsi :

(1) une villageoise qui lui faisait boire pour les poumons de l'eau de pluie au goût de résine $\left(\mathrm{F}^{14}\right)$ - 'wieśniaczka, która poiła go, na płuca, wodą deszczową o smaku żywicy',

(2) Je nous revois tous ensemble au lit, ma mère nous faisant boire de la bourrache (F) - 'Wyobrażam sobie znowu, jak wszyscy leżymy w łóżku, a matka poi nas ziółkami',

(3) elle nous fit boire une infusion (F) - 'napoiła nas herbatką ziołową',

(4) le philtre d'amour que la servante, par mégarde, leur a fait boire... (F) - napój miłosny, którym niechcący napoiła ich służąca...'.

Un cas qu'il faut mettre à part est faire boire utilisé avec $\mathrm{N}_{1}$ [alcool]. L'emploi de poić à l'imperfectif est possible, même si d'autres constructions verbales s'imposent parfois, telles par exemple dawać / dać $+\mathrm{N}_{\text {[liquide] }}$ ou dawać / dać do picia $+\mathrm{N}_{\text {[liquide] }}$, surtout quand on veut marquer que 'faire boire de l'alcool' n'est ni excessif, ni régulier, comme dans les exemples (5) - (7) ci-dessous :

(5) à l'époque, on jugeait sain de faire boire du vin aux enfants (F) - 'Sądzono wówczas, że zdrowo jest poić dzieci winem / dawać dzieciom (do picia) wino',

(6) Un barman [...] me faisait boire du cognac (F) - 'Barman [...] poił mnie koniakiem',

(7) il y a une méthode pour juger si le whisky est bon à être bu ou pas : on fait boire du whisky et on demande à celui qui vient de le boire... (F) - 'jest metoda, żeby sprawdzić, czy whisky jest dobra do picia, czy nie : ?poimy whisky / dajemy do (wy)picia whisky i pytamy tego, kto wypił...'

\footnotetext{
${ }^{14}$ Base textuelle Frantext, https://www.frantext.fr/.
} 
Il est à noter que pour les exemples $(5)$ - (7), il est possible de créer deux formes perfectives : napoić et spoić, mais le sens de chacune est différent. Napoić signifiera 'faire boire à sa soif' et spoić 'faire boire à l'excès'. Ainsi : il a fait boire du vin aux enfants - 'napoił / spoił dzieci winem', Un barman m'a fait boire du cognac - 'Barman napoił / spoił mnie koniakiem', On m'a fait boire du whisky - 'Napojono / spojono mnie whisky'.

\section{b. X fait boire $Y_{\text {[animé] }}$}

Poić/napoić s'utilise aussi avec le sujet non animé :

(8) il y avait un abreuvoir où ils [...] faisaient boire les cinq chevaux (F) - 'był wodopój, gdzie poili wszystkie pięć koni',

(9) C'est moi qui ai fait boire le veau tout à l'heure, dit Delphine (F) - 'to ja napoiłam przed chwilą cielaka - powiedziała Delphine'.

\section{c. Y fait boire $Y$}

Le même couple imperfectif/ perfectif polonais (poić/napoić) est utilisé quand faire boire apparaît dans son emploi absolu. Pourtant, d'autres formes peuvent être sélectionnées dans ce cas, notamment dawać / dać pić. Le complément non exprimé peut désigner toute espèce de boissons mais nous avons remarqué que ceux qui ne se réfèrent pas à l'alcool sont rares :

(10) ...comme on soutient une nuque, un ami alité qu'on veut faire boire... (F) - '... tak jak podtrzymuje się głowę chorego przyjaciela, którego chcemy ?napoić / któremu chcemy dać pić,

(11) Elle me fit boire, essaya de me donner un peu de bouillon froid, puis elle s'assit près de la fenêtre (F) - '?napoiła mnie / dała mi pić, spróbowała dać mi trochę zimnego bulionu, a potem usiadła przy oknie'.

L'emploi de poić / napoić oscille vers le sens 1 donné par $P W N$, soit 'donner à boire à quelqu'un jusqu'à assouvir sa soif', alors que dać pić sous-entend plutôt une petite quantité de liquide.

Concernant les emplois absolus où le complément non exprimé est un alcool, le verbe perfectif napoić semble exclu. En effet, il signifierait 'boire à sa soif'. Par contre, spoić kogoć ('faire boire quelqu'un jusqu'à le soûler') est acceptable, à côté des autres solutions comme dać (komuś) alkoholu / wódki :

(12) Il avait voulu faire boire l'enfant, qui s'y était refusée (F) - 'Chciał ją *napoić / dać jej alkoholu / wódki, ale dziewczynka odmówiła',

(13) l'homme, qu'on essayait de faire boire pour le faire changer d'avis (F) - 'mężczyzna, którego usiłowaliśmy *napoić / spoić, żeby zmienił zdanie',

(14) Alors j'ouvrirais la bouteille de Champagne, pour le faire boire (F) - 'Otworzyłbym butelkę szampana, *żeby go napoić / żeby go spoić / ?żeby (sobie) wypił’. 


\section{d. faire boire + quant. $+\mathrm{N}_{1[\text { liquide] }}$}

L'ajout après faire boire d'un quantitatif bloque l'emploi de poić / napoić / spoić en polonais. La solution la plus naturelle est alors de traduire la construction causative du français par dawać / dać (wy)pić / do (wy)picia) ('donner qqc. à boire). Les quantitatifs qui apparaissent se réfèrent soit à une quantité assez précise, comme par exemple un verre, un demi-verre, une gorgée, une goutte, le reste, un bol:

(15) Lalla lui fait boire un verre d'eau (F) - 'Lalla daje jej / mu (wypić / do (wy)picia) szklankę wody' (*poi ją / go szklanką wody),

(16) notre mère [...] me fait boire un bol de bouillon (F) - 'nasza matka [...] daje mi (do (wy) picia) kubek bulionu' (?poi mnie kubkiem bulionu),

(17) Puis il me fit boire trois gorgées de la mixture (F) - 'Potem dał mi (do wypicia) trzy łyki mikstury' (*napoił mnie trzema łykami mikstury),

soit à une quantité imprécise :

(18) Tu me fais boire trop de vin blanc (F) - 'Dajesz mi za dużo białego wina'.

Après avoir vérifié s'il existe, dans le corpus $N K J P^{15}$, des emplois du verbe poić avec les expressions de quantité, nous avons constaté un manque total d'occurrences pour poić + kubek (un bol), poić + kieliszek (un verre à pied), poić + tyk (une gorgée) et poić + kropla (une goutte). Nous avons trouvé une seule occurrence pour poić + szklanka (un verre) :

(19) każesz go może nawet poić szklanka mleka (NKJP) - 'tu lui fais peut-être boire un verre de lait',

et une pour poić + butelka, mais ce syntagme a un sens particulier, figé, et signifie 'donner un biberon à un bébé' :

(20) Może pojenie butelka oducza małego ssania piersi (NKJP) - 'Il est possible que donner un biberon fait oublier le sein au bébé'.

\section{e. faire boire $+\mathbf{N}_{1[\text { métonymiel }}$}

Le blocage de poić / napoić / spoić est encore plus fort quand faire boire est utilisé par métonymie avec $\mathrm{N}_{1[q u a n t i t a t i f]}$ pour parler de la substance, du liquide, la métonymie étant « la figure consistant à remplacer un nom par un autre nom en raison d'un rapport qui lie les référents de l'un et de l'autre dans la réalité $\rangle^{16}$. Polguère ${ }^{17}$ parle dans ce cas de la contiguité des concepts et dit qu'« une lexie L2 est liée par un lien

${ }^{15}$ Narodowy Korpus Języka Polskiego, http://nkjp.pl/.

${ }_{16}$ M. Riegel, op. cit., p. 954.

17 A. Polguère, Lexicologie et sémantique lexicale, Montréal, Les Presses de l'Université de Montréal, 2008, p. 198. 
sémantique de métonymie à un copolysème $\mathrm{L} 1$ si elle dénote un concept qui est perçu comme contigu au concept dénoté par L1 ». En effet, alors que le verbe polonais pić se construit avec des quantitatifs par métonymie comme correspondant de boire : pić / wypić szklaneczke, kieliszek, filiżanke, kubek, butelke ('boire un verre, une tasse, un bol, une bouteille'), poić / napoić / spoić s'avère impossible avec les expressions de quantité : *(na)poić szklaneczka, kieliszkiem, filiżanka, kubkiem, butelka ('faire boire un verre, une tasse, un bol, une bouteille'). La solution qui reste est la traduction par dawać/dać (do wypicia) ou bien par un verbe non causatif wypić :

(21) Pour le calmer, on lui fit boire le reste de la bouteille (F) - 'Żeby go uspokoić, daliśmy mu (do wypicia) resztę butelki',

(22) La bouteille que t'as fait boire à Doudou (F) - 'Butelka, którą wypiła Doudou / Butelka, którą dałeś Doudou (do wypicia)',

(23) mais je l'en supplie, qu'il ne me serve pas le verre qu'il veut me faire boire ! (F) - 'Ale błagam go, niech nie podaje mi szklanki, którą mam wypić / którą chce, żebym wypił’.

\section{f. $\quad \mathrm{N}_{0 \text { [inanimé] }}+$ se faire boire}

Le verbe boire dans le sens d'absorber un liquide' (TLFi), 's'imbiber, s'imprégner' $(L V F)$ peut entrer dans une construction causative se faire boire. Mais, alors que boire avec le sujet inanimé peut correspondre à pić en polonais : Le buvard boit l'encre - 'Bibuła pije wodę' (sans complément exprimé, ceci n'est plus évident : Cette éponge boit bien - 'Ta gąbka dużo ?pije'), la construction factitive ne se traduira pas en polonais par poić, mais par wsiąkać, qui n'est pas un verbe causatif :

(24) Quelques gouttes dévalent le long du goulot et viennent se faire boire par le bois blanc (F) - 'Kilka kropli spływa z butelki i wsiąka w białe drewno'.

\section{Conclusion}

1) Des conclusions qui s'imposent, l'essentielle concerne l'emploi de faire boire avec un quantitatif. En effet, ce dernier bloque en polonais le verbe (-)poić, alors qu'en français, il est tout à fait naturel de l'utiliser avec faire boire. Ainsi, la construction $\mathrm{X}+$ faire boire $+\mathrm{N}_{1[\text { [iquide] }}$ à $\mathrm{Y}$, correspond à $\mathrm{X}+(-)$ poić $+\mathrm{Y}+$ $\mathrm{N}_{1[\text { liquide }]}$ :

tu me fais boire du vin blanc - poisz mnie biatym winem

elle lui fait boire du café - poi ja / go kawa

mais l'ajout d'un quantitatif bloque automatiquement (-)poić :

tu me fais boire une gorgée de vin blanc

*poisz mnie tykiem biatego wina

dajesz mi łyk białego wina 
elle lui fait boire une tasse de café

*poi ja / go filizanka kawy

daje jej / mu filiżankę kawy

Bien sûr, le même blocage intervient dans les cas d'emploi métonymique, car un quantitatif est utilisé par métonymie comme $\mathrm{N}_{1[\text { liquide] }}$.

2) Le couple des verbes causatifs polonais poić / napoić sert à traduire faire boire avant tout dans l'emploi que nous pourrions appeler 'prototypique', c'est-àdire quand faire boire s'utilise avec $\mathrm{N}_{1[\text { liquide] }}$ : $\mathrm{X}+$ faire boire $+\mathrm{N}_{1[\text { [iquide] }}+$ à $\mathrm{Y} \rightarrow \mathrm{X}$ + poić / napoić $+\mathrm{Y}+\mathrm{N}_{1[\text { liquide }]}$, soit :

Luc fait boire de l'eau à Marc-Luc poi Marca woda

Luc a fait boire de l'eau à Marc - Luc napoit Marca woda

3) L'exception est faite de cette règle quand $\mathrm{N}_{1[\text { liquide] }}$ est un alcool. À l'imperfectif, poić s'utilise à côté de dawać (do (wy)picia). Au perfectif, la situation se complique et fait apparaître deux sens distincts, l'un marqué fortement de la propriété 'excès'. À ces deux sens correspondront deux verbes préfixés, napoić (ou dać do (wy)picia) et spoić :

Luc fait boire du vin à Marc $\rightarrow$

Luc a fait boire du vin à Marc $\rightarrow$
Luc poi Marca winem / Luc daje Marcowi wina Luc napoit Marca winem [sans excès]

Luc spoit Marca winem [excès]

Ainsi :

$$
\begin{aligned}
& \mathrm{N}_{\text {o[humain] }}+\text { faire boire }+\mathrm{N}_{1[\text { liquide, non alcool] }} \rightarrow \text { poić / napoić } \\
& \mathrm{N}_{\text {o[humain] }}+\text { faire boire }+\mathrm{N}_{1[\text { [alcool] }} \rightarrow \text { poić / napoić, dawać / dać do picia [sans excès] } \\
& \text { poić / spoić [excès] }
\end{aligned}
$$

Utilisé dans son emploi absolu, faire boire se traduit en polonais par les mêmes verbes, suivant qu'il y a une idée d'excès ou non.

4) Faire boire utilisé avec $\mathrm{N}_{\text {o[animé non humain] }}$ se fait correspondre en polonais poić / napoić, comme son emploi prototypique :

Luc fait boire les chevaux $\rightarrow$ Luc poi konie

Luc a fait boire les chevaux $\rightarrow$ Luc napoit konie

soit :

$$
\mathrm{N}_{0[\text { humain] }}+\text { faire boire }+\mathrm{N}_{1[\text { [animé non humain] }} \rightarrow \mathrm{N}_{0[\text { hhumain] }}+\text { poić / napoić }+\mathrm{N}_{1[\text { animé non humain] }}
$$

5) Enfin, à la construction causative passive se faire boire correspond en polonais le verbe non causatif wsiakać / wsiaknać. 


\section{Bibliographie}

Base textuelle Frantext, https://www.frantext.fr/

Bat-Zeev Shyldkrot, Hava, "Présentation. Les auxiliaires : délimitation, grammaticalisation et analyse », Langages, 1999, $\mathrm{n}^{\circ}$ 135, Paris, Armand Colin, p. 3-7

Ducrot, Oswald et Schaeffer, Jean-Marie, Nouveau dictionnaire encyclopédique des sciences du langage, Paris, Editions du Seuil, 1995

Grevisse, Maurice, Le Bon usage, Louvain-la-Neuve, Duculot, 1993

Gross, Maurice, «Les bases empiriques de la notion de prédicat sémantique », in Langages, 1981, $\mathrm{n}^{\circ}$ 63, Paris, Armand Colin, p. 7-52

Les Verbes français de Jean Dubois et Françoise Dubois-Charlier (Version LVF+1), http://rali.iro. umontreal.ca/rali/?q=fr/lvf

Narodowy Korpus Języka Polskiego, http://nkjp.pl/

Polguère, Alain, Lexicologie et sémantique lexicale, Montréal, Les Presses de l'Université de Montréal, 2008

Ponchon, Thierry, Sémantique lexicale et sémantique grammaticale : le verbe 'faire' en français médiéval, Genève, Librairie DROZ S.A., 1994

Riegel, Martin, Grammaire méthodique du français, Paris, Presses Universitaires de France, 1994

Słownik Języka Polskiego PWN, https://sjp.pwn.pl/

Stosic, Dejan, «Le rôle des préfixes dans l'expression du déplacement. Eléments d'analyse à partir des données du serbo-croate et du français », Cahiers de Grammaire, Toulouse, Université de Toulouse-le-Mirail, 2001, n 26, p. 207-228, <halshs-00272879>, consulté le 21.05.2018

Trésor de la Langue Française informatisé, http://atilf.atilf.fr/

Wielki Stownik Języka Polskiego, http://www.wsjp.p1/

Zatorska, Agnieszka, " Z problematyki polskich i słoweńskich analityzmów kauzatywnych z parafrazą przymiotnikową », Studia z Filologii Polskiej i Stowiańskiej, Warszawa, Instytut Slawistyki Polskiej Akademii Nauk, 2015, nº 50, p. 237-248

Joanna Cholewa - Maître de conférences à l'Université de Białystok (Pologne), HDR en linguistique, auteure de deux monographies et de nombreux articles. Ses travaux de recherche s'inscrivent dans le domaine de la sémantique, et portent essentiellement sur la conceptualisation et l'expression du mouvement et des relations spatiales. 